\title{
Lavaux: plan de protection - plan d'exception
}

\section{Introduction}

Le texte qui va suivre surprendra, voire décevra plus d'un géographe par son manque d'orthodoxie. Plutôt que de centrer l'analyse sur les composantes géographiques du paysage déterminantes pour la politique de protection, l'auteur a préféré présenter les divers moments forts de l'histoire d'une société qui, après avoir été aux prises avec son espace vital et l'avoir maîtrisé, s'en voit tout à coup ravir partiellement le contrôle, au nom de la protection du paysage.

Originaire de Lavaux, travaillant depuis une quinzaine d'années au Service de l'aménagement du territoire, l'auteur, en tant que député, a eu par ailleurs à se prononcer sur la loi présentée en fin d'article; c'est dire qu'il a été mêlé a plus d'un titre au sujet et que l'objectivité scientifique lui était difficilement accessible.

Que le lecteur lui pardonne dès lors d'avoir cédé à une interprétation qui doit autant à l'approche intuitive qu'à la démarche géographique traditionnelle.

\section{Lavaux: De l'organisation du pays viticole a sa récente détérioration}

\section{Le cadrage géographique de la région}

Lavaux, en dépit de son nom, est un coteau. Il représente la partie supérieure d'un versant de vallée dont le fond se trouve noyé par le lac Léman. Sa forte pente (elle passe de 13 à $43 \%$ d'ouest en est, ONDE 1971) et son orientation sud-sud-ouest le désignèrent dès le Moyen Age comme une zone de culture privilégiée, gratifiée de la réverbération du lac et de son rôle de volant thermique.

Si sa frange inférieure, constituée par le niveau du Léman (372 mètres), est nette, sa limite supérieure apparaît irrégulière, ligne festonnée où alternent vallonements et collines de molasse ou de poudingue, tels que le Signal de Grandvaux (769 m), la Tour de Gourze $(927 \mathrm{~m})$, les Monts de Cheseaux $(982 \mathrm{~m})$ et le Mont Pélerin (1080 m). Dès l'altitude de 600 mètres, le vignoble fait place aux prairies et aux forêts. Les frontières latérales de Lavaux à l'ouest, du côté de l'agglomération lausannoise, comme à l'est, du côté de Vevey sont nettement définies par deux rivières, la Paudèze et la Veveyse, qui pour l'une entaille profondément le soubassement molassique et pour l'autre crée la ligne de transition entre le Moyen-Pays et les Préalpes.

Lavaux occupe ainsi une bonne moitié de l'adret lémanique oriental (voir fig. 1). Sa superficie, les communes de Chardonne, Corseaux, Corsier, Jongny comprises, est de 14000 hectares environ, dont 1000 sont en vigne.

\section{De l'aménagement du vignoble de Lavaux à l'organisation régionale}

Confrontés à ces conditions naturelles climatiquement favorables mais exigeantes quant à la mise en valeur des terroirs, les moines, aidés bientôt par des cohortes de bâtisseurs et de vignerons, allaient mener une gigantesque action d'aménagement forçant la nature dans ses éléments les plus hostiles.

Grâce au génie architectural collectif, le relief deviendra construction. Les murs horizontaux ou légèrement obliques composeront avec les bancs de molasse, de poudingue et viendront marquer les zones de transition, les lignes d'inflexion entre les évidements et les zones d'accumulation. Les tracés des murs verticaux eux, seront commandés par les épaulements, les arrêtes, les ravins. Les cheminements des eaux lors des plus fortes pluies deviendront des coulisses, ces collecteurs d'eau à ciel ouvert qui souligneront encore le quadrillage. Toute l'architecture procède d'une intimité exceptionnelle avec la nature, d'une connaissance inspirée et profonde de ses éléments, de la conviction partagée de mener une grande ouvre à la gloire de Dieu dans un mouvement panthéiste.

A l'aménagement du paysage va s'ajouter le plan d'urbanisation selon les principes suivants:

$A u$ bord du lac, sur la voie est-ouest, les bourgs de Lutry et Cully avec leurs églises, leurs fortifications, leurs ports, leurs halles et leur bourgeoisie naissante constituée d'artisans et de commerçants.

Victor Ruffy, Géographe, Service de l'aménagement du territoire, Case postale, 1000 Lausanne 17. 


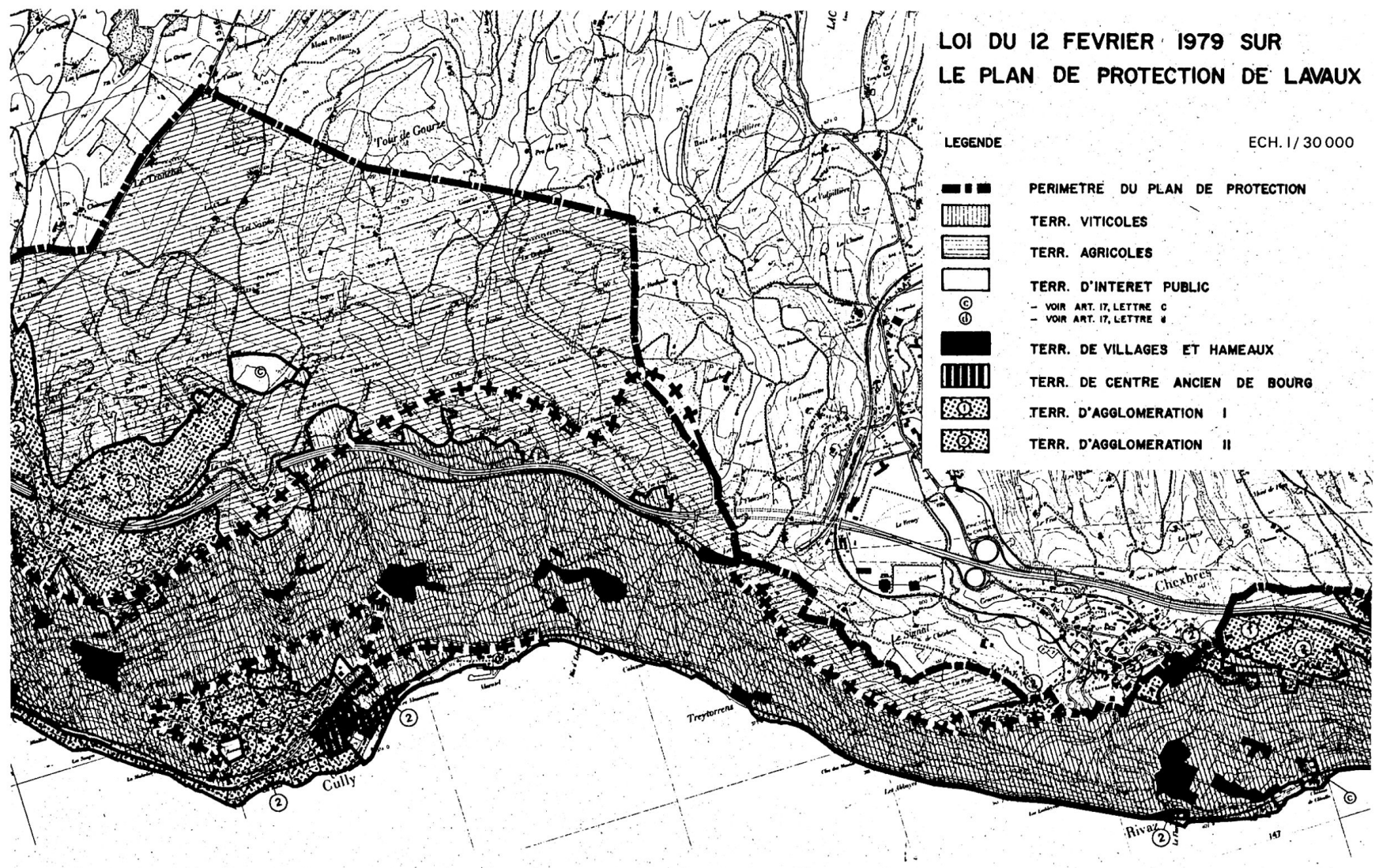

Fig. 1 EXTRAIT DU PLAN

$+\mathbf{+}$ PERIMETRE OU TERRITOIRE A PROTEGER PREVU PAR L'INUENTAIRE DES PAYS
DE 1967 


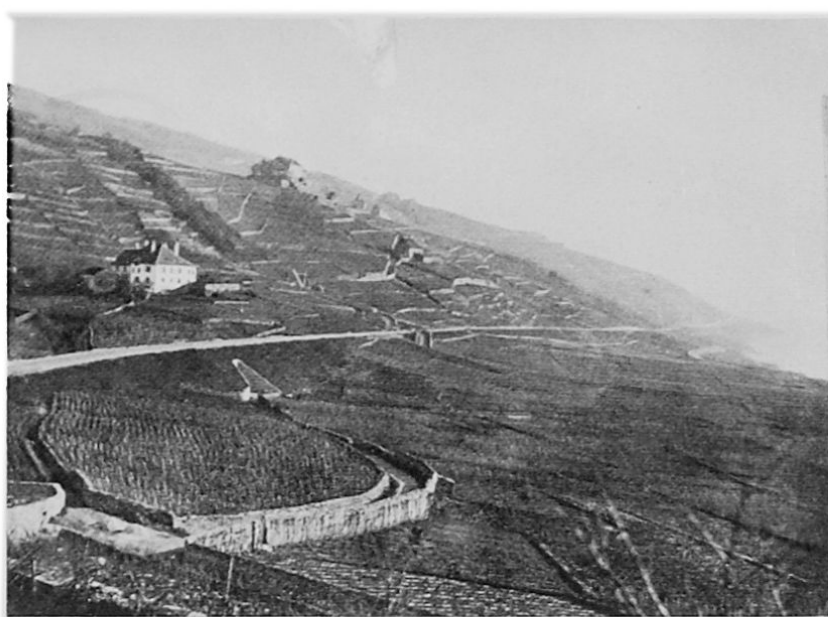

Photo 1: Environ 1900

$A$ mi-côte, les villages et hameaux exclusivement vignerons, Grandvaux, Riex, Epesses, Corsy, Savuit, Aran.

Sur les Monts, les fermes de colonisation, plus tardives, mais indispensables pour assurer la complémentarité de l'économie viticole. Il leur appartenait en effet de fournir le bois pour les tonneaux et les échalas, la paille pour attacher la vigne et de ravitailler la population en viande, en lait, dans la mesure où la progression implacable de la vigne reléguait obligatoirement l'élevage vers les Hauts.

\section{De la culture de la vigne au paysage symbolique}

Les soins portés à la plante et aux cultures furent à l'égal de ceux prodigués à la création du terroir. La recherche de l'adaption parfaite d'un cépage au milieu naturel a duré des décennies, voire des siècles. Stimulés par la Confrérie des vignerons, les efforts ont porté sur le mode de sélection, par chaponnage, la taille, de type courte sur charpente courte, les travaux de la feuille, répétés trois fois l'an et l'écartement entre les plants, habituellement d'un mètre. Plaçant très haut l'art de cultiver la vigne et plus spécialement le Chasselas, cépage probablement autochtone, témoignant de la précocité du caractère intensif des cultures, ces préoccupations sont venues parfaire l'extraordinaire géométrie du paysage par l'introduction des cultures en lignes orientées dans le sens de la pente.

La beauté construite du paysage, faite de rigueur et de pureté, sa précision, son homogénéité, le contraste avec les espaces limitrophes, tout concourt à l'éclat de cette performance sociale.

Le paysage est devenu symbole de persévérance, de victoire sur le milieu naturel, de maintien d'une tradition.

Les vignerons d'aujourd'hui, le plus souvent à la tête d'expoitations familiales, gardant pour la plupart d'entre eux la maîtrise de leur production

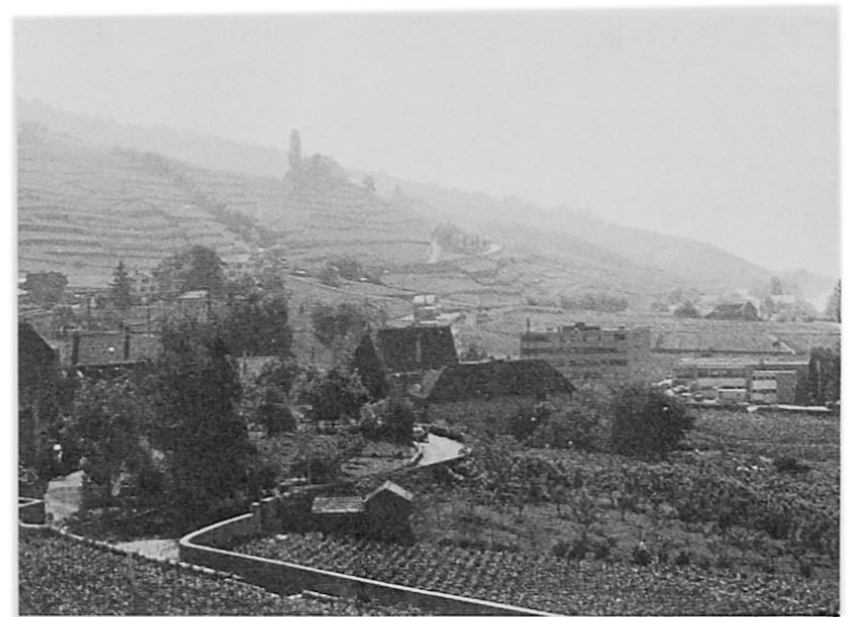

Photo 2: 1979

jusqu'à la phase ultime qu'est la commercialisation de la bouteille, en sont parfaitement conscients.

\section{L'urbanisation des XIXème et XXème siècles dans le canton de Vaud et à Lavaux}

Au cours des XIXème et XXème siècle, le phénomène d'urbanisation se répandit d'une façon très différente suivant les régions du canton. Lausanne qui comptaint 9965 habitants en 1803, en abritait 47000 en 1900 et en dénombre 136000 en 1976. Le développement du croissant lémanique s'affirmait par rapport au reste du canton.

La création des chemins de fer Lausanne-Berne, Lausanne-Brigue, Puidoux-Vevey, les lignes de tramways puis de trolleybus, assurant la liaison Lausanne-Lutry et Lausanne-Savigny, diminuaient singulièrement la distance-temps séparant le coeur de Lausanne des communes périphériques. Les conditions permettant à la fonction résidentielle de migrer à l'extérieur étaient désormais réunies et le processus de déversement à partir de Lausanne était amorcé. Une évolution identique se produisait sur la frange orientale du vignoble mais avec une intensité moindre en raison de l'extension plus lente de Vevey.

Dès lors, le grignotage du vignoble se développera sur ses flancs et le long des voies de communication (chemin de fer et route), c'est-à-dire le long du lac et sur la route des Monts entre le vignoble et les collines (voir photographies 1 et 2).

«La construction suit son cours, surtout entre la ligne de chemin de fer Lausanne-St-Maurice et le lac; dans un avenir prochain, les vignes actuelles seront remplacées par de jolies villas. De ce fait les terrains ont plus que doublé de valeur» (FOSCALE 1917).

Aux causes économiques externes et notamment à la rente foncière bien supérieure accordée à la production de logements qu'à celle du vin, il faut en 
ajouter d'autres qui sont directement liées à l'economie viticole. La succession de mauvaises années entre 1909 et 1918 a déclenché une régression rapide du vignoble; celui de Lutry passe par exemple durant cette période de 260 à 216 hectares (voir tableau 1).

\section{Le minage du vignoble par l'intérieur}

La phase récente de l'urbanisation a touché le coeur même du vignoble. Dès 1960, l'extension urbaine est non seulement en passe de phagociter la moitié ouest de la commune de Lutry, mais amorce une percée en force au centre du vignoble à partir de Cully (voir tableau 2).

Gravissant la pente, l'urbanisation emprunte les anciennes routes qui reliaient les populations du bas avec celles du haut dans le cadre de l'écomomie ancienne et, sous forme de villas et immeubles locatifs, elle opère dans un premier temps la jonction entre Cully et Grandvaux-village (voir photographie de la couverture) et entre Cully et Riex. Dans un second temps, elle se prolonge entre Grandvauxvillage et Grandvaux-gare d'un côté et entre Riex et Chenaux de l'autre. A partir de ce moment-là, environ vers 1960, le vignoble de Lavaux est scindé en deux avec une partie relativement intacte à l'est de l'axe Cully-Riex et une autre moins homogène, sorte de poche vulnérable dans laquelle les perspectives de plus-value sur les vignes excitent l'appétit des promoteurs et démobilisent progressivement les vignerons (JAQUES 1970).

\section{Surface totale}

\begin{tabular}{lll}
\hline Canton de Vaud & District de Lavaux & $\%$ du canton \\
321900 ha & 12221 ha & 3,80 \\
\hline
\end{tabular}

Surface du vignoble en hectares

\begin{tabular}{llllllll}
\hline \multicolumn{2}{l}{ Canton de Vaud } & & District de Lavaux & Lutry & Grandvaux & Cully & Corseaux \\
\hline 1909 & 6699 & 655 & 9,78 & 260 & 79 & 97 & 55 \\
1918 & 4654 & 697 & 14,98 & 216 & 81 & 88 & 44 \\
1950 & 3691 & 609 & 16,50 & 177 & 67 & 73 & 27 \\
1960 & 3475 & 593 & 17,06 & 167 & 64 & 70 & 27 \\
1970 & 3299 & 565 & 17,13 & 148 & 61 & 68 & 24 \\
\hline
\end{tabular}

Tableau 1: Evolution du vignoble vaudois, de Lavaux et des communes particulièrement touchées par la construction.

Mises a l'enquête

\begin{tabular}{|c|c|c|c|c|c|c|c|c|c|}
\hline & \multicolumn{3}{|c|}{ Habitations collectives } & \multicolumn{3}{|c|}{ Maisons familiales } & \multirow{2}{*}{$\begin{array}{l}\text { Population } \\
\text { Canton }\end{array}$} & \multirow[b]{2}{*}{ Lavaux } & \multirow[b]{2}{*}{$\%$ de Vaud } \\
\hline & Canton & Lavaux & $\%$ de Vaud & Canton & Lavaux & $\%$ de Vaud & & & \\
\hline 1965 & 344 & 17 & 4,94 & 695 & 54 & 7,77 & 480914 & 13199 & 2,74 \\
\hline 1966 & 361 & 12 & 3,32 & 769 & 54 & 7,02 & 486501 & 13670 & 2,81 \\
\hline 1967 & 258 & 7 & 2,71 & 752 & 69 & 9,18 & 494746 & 13778 & 2,78 \\
\hline 1968 & 285 & 9 & 3,16 & 815 & 50 & 6,13 & 500068 & 13993 & 2,80 \\
\hline 1969 & 307 & 16 & 5,21 & 1052 & 65 & 6,18 & 506656 & 14325 & 2,83 \\
\hline 1970 & 294 & 9 & 3,06 & 1029 & 59 & 5,73 & 511966 & 14801 & 2,89 \\
\hline 1971 & 368 & 26 & 7,07 & 1106 & 92 & 8.32 & 515904 & 15101 & 2,93 \\
\hline 1972 & 403 & 25 & 6,20 & 1559 & 100 & 6,41 & 522408 & 15489 & 2,96 \\
\hline 1973 & 242 & 18 & 7,44 & 1217 & 77 & 6,33 & 522798 & 15850 & 3,03 \\
\hline 1974 & 281 & 27 & 9,61 & 1152 & 104 & 9,03 & 526828 & 16150 & 3,07 \\
\hline 1975 & 201 & 10 & 4,98 & 634 & 55 & 8,68 & 525864 & 16450 & 3,13 \\
\hline 1976 & 155 & 10 & 6,45 & 868 & 59 & 6,80 & 523885 & 16614 & 3,17 \\
\hline 1977 & 175 & 13 & 7,43 & 1022 & 71 & 6,95 & 524319 & 16787 & 3,20 \\
\hline
\end{tabular}

Tableau 2: Evolution de la construction dans le canton et à Lavaux.

Publication de l'Office vaudois pour le développement du commerce et de l'industrie. (Economie Vaudoise 1975-1976) 


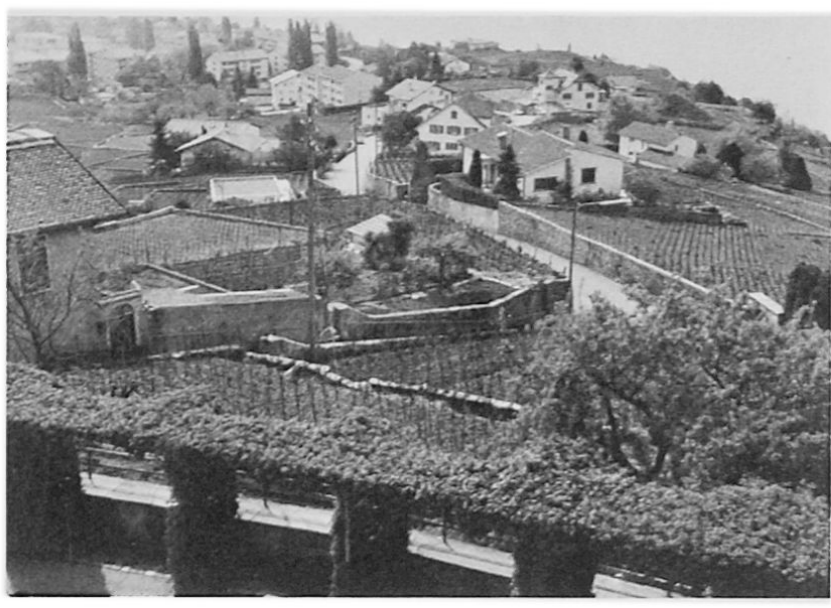

Phase récente d'urbanisation du vignoble de Lavaux entre Cully et Grandvaux.

\section{De la politique des petits pas au nouvel article constitutionnel}

\section{Les premières mesures de protection}

Même si le recul massif du vignoble date des années 1960 , on a peine à croire qu'aucune tentative n'ait été entreprise en vue de préserver Lavaux d'une urbanisation désordonnée et prévisible. L'historique des interventions montrant l'écart séparant les moyens à disposition des objectifs à atteindre reste à faire. Nous nous bornerons pour cette raison à ne mentionner que les démarches dont nous avons connaissance. Il fut un temps où le tracé des routes et surtout les abords de ces dernières étaient considérés comme très importants au point de vue esthétique par le bureau du plan d'extension cantonal, l'ancêtre du service de l'aménagement du territoire. La route du bord du lac entre Lausanne et Vevey ne devait pas échapper à ce souci. On doit à PAUL BUDRY et JEAN-PIERRE VOUGA l'aménagement des talus bordant le tronçon de Cully à Vevey. Les cytises et les cyprès qui agrémentent la vue depuis la route sont le résultat d'un plan élaboré durant la deuxième guerre mondiale à la suite d'une excursion faite en voiture circulant alors au charbon de bois. D'autres mesures, pour la plupart ponctuelles comme la protection des toits de St-Saphorin ou encore l'achat de parcelles particulièrement vulnérables sur la Tour de Gourze, témoignent du cas que l'on faisait de ce paysage, de tout temps cher aux Vaudois; mais l'intervention la plus nette tant dans ses objectifs que dans ses mesures fut sans conteste l'adoption en 1950 du plan d'extension cantonal du Dézaley, mettant sous protection dans le coeur de Lavaux les parchets de son plus prestigieux terroir. L'authentique sauvegarde cantonale du vignoble devait pourtant en rester là malgré les efforts renouvelés de JEAN-PIERRE VOUGA, devenu architecte cantonal.
Dès 1964 , la loi vaudoise sur la police des constructions, profondément remaniée et complétée par un volet consacré à l'aménagement du territoire, offrait aux communes la possibilité de décréter des zones agricoles ou viticoles dans lesquelles les constructions de villas ou de bâtiments locatifs seraient désormais exclues. Mises devant leur propre responsabilité, les communes agirent diversement. Alors que certaines d'entre elles tirèrent parti rapidement de cette nouvelle base légale, nous pensons notamment à Lavaux à celle d'Epesses, d'autres, les plus engagées dans l'urbanisation, craignant des difficultés, choisirent la temporisation et continuèrent de subir la loi de la construction désordonnée. La loi cantonale sur la protection de la nature, des monuments et des sites entrait en force en 1969. L'inventaire de 1971, qui la complétait, réaffirmait la volonté politique de protéger ce site au niveau du canton.

Enfin, l'acuité des problèmes de Lavaux, l'importance de l'aménagement pour cette région ne furent pas étrangères au choix de l'Office de l'urbanisme qui en fit le cadre de son premier plan directeur régional. En négligeant d'associer étroitement dès le départ et régulièrement les autorités des communes concernées, les auteurs orientèrent leur travail sur une analyse technique un peu mécaniste, théorique et se privèrent de la possibilité de traiter la phase capitale de la mise en oeuvre du programme proposé. La publication du document (A. BYRN 1971) provoqua le raidissement des Municipalités, qui refusèrent d'entrer en discussion sur une telle base, créèrent la Commission intercommunale d'urbanisme de Lavaux (CIUL) et chargèrent un urbaniste privé de refaire un plan directeur régional pour leur compte (URECH 1977).

\section{Le plan de Villette et les raisins de la colère}

Ce fut, à la surprise générale, l'une des communes de Lavaux les mieux disposées à l'égard des mesures de protection qui déclencha la création du mouvement "Sauver Lavaux». On était à la veille de l'arrêté fédéral introduisant des mesures urgentes en matière d'aménagement, qui allait permettre de geler provisoirement la situation.

Nous ne retiendrons de l'historique de l'affaire de Villette dont P.A.DUMMER a fait une analyse très complète que les éléments résumés expliquant l'apparition du mouvement «Sauver Lavaux» (DUMMER 1973). Voulant sauvegarder la majeure partie de son vignoble, aucunement protégé par le plan alors en vigueur, la Municipalité de cette commune, avec l'appui des autorités cantonales, prit l'initiative de le modifier. Désireuse d'introduire une zone viticole inconstructible, elle proposa pour y parvenir le regroupement de très nombreux droits à bâtir existants et dispersés dans le prolongement du village 
actuel. Afin d'avoir barre sur le style d'architecture, la réalisation de ces droits à bâtir devait être subordonnée à un plan de quartier. Il s'agissait d'un des cas les plus complets de péréquation réelle opéré dans le canton de Vaud et recourant à toutes les possibilités du cadre légal en vigueur.

Toutefois, déplorant l'importance des constructions (43 villas contiguës), critiquant l'empiétement sur les terres en vigne les plus faciles à travailler, un mouvement d'opposition prit corps, déclarant toute nouvelle construction dans les vignes comme inutile, donc injustifiée. Faisant peu cas de la situation juridique préexistante, ignorant délibérément le poids des institutions en place, le mouvement «Sauver Lavaux» présidé par F. WEBER part en campagne sans se préoccuper des intérêts fonciers qui sont en jeu.

Entouré d'un comité comprenant des vignerons, des résidents de Lavaux, des journalistes et des artistes, F. WEBER en appelle d'abord à la population suisse au moyen d'une pétition et ensuite à la population vaudoise en lançant une initiative constitutionnelle.

"La région de Lavaux, de la Lutrive à Corsier, est déclarée site protégé. La loi détermine l'exécution de cette disposition».

Aux côtes de F. WEBER se rangèrent les défenseurs du visage aimé de la patrie et plus spécialement les amoureux de Lavaux, des vignerons convaincus de la nécessité de protéger leur profession, des résidents plus ou moins égoïstes aussi et beaucoup de citoyens désireux de troubler le jeu de la spéculation foncière. Dans le camp opposé se regroupait la majorité des autorités politiques respectueuses des institutions, accordant leur crédit aux moyens déjà à disposition, des vignerons désireux de rester maîtres chez eux, des citoyens redoutant le caractère sommaire et exceptionnel de la mesure, des représentants des milieux immobiliers.

L'ordre des valeurs mis en avant par celui qu'on a tenté de disqualifier en le traitant d'étranger, permit incontestablement à un malaise latent dans de nombreuses couches de la population de s'exprimer et de se traduire par une mesure concrète.

Afin de contrer l'initiative, le Conseil d'Etat élabora un contre-projet sous la forme d'un article constitutionnel de portée générale et ainsi rédigé: «Les paysages, les sites, les monuments et les localités qui méritent d'être sauvegardés en raison de leur intérêt esthétique, historique, scientifique ou éducatif sont protégés par la loi».

Il fut approuvé par la majorité du Grand Conseil. Dans un préavis motivé, le Grand Conseil invita le peuple vaudois à refuser l'article proposé par F. WEBER au profit de cet article officiel.

Malgré ça, le 12 juin 1977, le peuple vaudois acceptait l'initiative "Sauver Lavaux» par 64824 voix contre 49217 et repoussait le contre-projet du
Grand Conseil par 61486 voix contre 39618. (A Lavaux les chiffres respectifs furent les suivants: Refus de l'initiative par 2801 non contre 2266 oui et refus du contreprojet par 2649 non contre 2022 oui).

\section{La loi sur le plan de protection}

\section{Introduction}

$\mathrm{Au}$ lendemain du scrutin, le Conseil d'Etat se trouva donc contre son gré dans l'obligation de préparer une loi assurant la protection de Lavaux jugée implicitement insuffisante dans le cadre de la législation existante. La tâche la plus délicate consista à concilier une interprétation respectant cette nouvelle volonté populaire avec une procédure permettant d'associer les communes à la mise sur pied du nouveau régime juridique applicable (Exposé des motifs de la loi du 13 décembre 1977). Cette dernière préoccupation devait conduire le Conseil d'Etat à écarter la solution du plan d'extension cantonal, qui une fois adopté dans son principe par le Grand Conseil, aurait été de sa compétence exclusive.

Il préféra l'élaboration d'une loi-cadre accompagnée d'un plan de protection. Recourant à l'urbaniste privé, qui était sur le point d'achever pour le compte des communes le plan directeur régional de Lavaux, le Conseil d' Etat concrétisait et sa volonté de faire participer les autorités locales et celle d'aller rapidement en besogne.

Que la première loi cadre du 13 décembre 1977 indiquant l'esprit général de la démarche et les principaux éléments de la systématique ait été abrogée pour des raisons formelles par celle du 12 février 1979 importe peu en l'occurrence. Il convient plutôt de voir qu'elles sont les caractéristiques du document définitif.

\section{L'esprit de la loi du 12 février 1979}

Le but général poursuivi par la loi apparaît dans l'exposé des motifs. "Le projet vise à une protection globale, répondant à des objectifs de protection multiples, dont l'ensemble doit être garant de l'identité de Lavaux".

La formule très générale est intéressante dans la mesure où elle va révéler quels sont les objets constitutifs de l'identité de Lavaux et quelle va être précisément le champ d'application de cette loi qui concrétise le mandat constitutionnel.

On ne sera pas surpris de voir apparaitre en premier lieu le maintien du vignoble en tant que composante fondamentale du paysage. La loi veut «garantir à long terme la continuité de la surface plantée en vigne de la Lutrive à Corsier, sur une surface d'une 
largeur suffisante pour être dominante dans le site».

La protection sera étendue à la région agricole des Monts dans la mesure où leurs versants s'inscrivent dans le prolongement direct du vignoble. Les pentes du Signal de Grandvaux et de la Tour de Gourze seront considérées comme partie intégrante du site de Lavaux, mais pas celles du Mont Pélerin, pourtant initialement englobées dans le périmètre provisoire.

La sauvegarde du site doit comprendre également le patrimoine architectural. Les hameaux, les villages, les centres des bourgs anciens tels Cully et Lutry doivent conserver leur silhouette. La volumétrie et le caractère des bâtiments seront protégés. Les volumes existants pourront être utilisés dans la mesure où cela ne nuit pas au caractère des bâtiments. Les activités nouvelles qui pourraient compromettre la vie d'un village viticole ou le caractère d'un centre ancien sont interdites.

Enfin, suivant leur situation, les territoires déjà construits ou destinés à l'être seront régis par des principes se rapportant entre autres à l'implantation au nombre de niveaux, deux ou trois, et à l'arborisation.

Afin d'assurer à la région des secteurs se prêtant à l'aménagement ou à la construction d'équipements d'intérêt général, il a été prévu des territoires d'intérêt public.

Le plan de protection accompagnant la loi (voir fig. 1) précise le périmètre général de protection ainsi que celui des divers territoires. Ces derniers seront désormais contraignants pour les communes qui, sous réserve de légères adaptations, devront les reprendre lors de la révision de leur plan de zones. Les délais impartis aux communes pour l'adoption de leur nouveau plan sont de deux ans. Durant cette période transitoire, tout permis de construire devra faire l'objet d'une autorisation préalable du Département des Travaux publics.

\section{Conclusions}

\section{La commune en question}

A travers la défaite infligée à l'aménagement officiel et le coup porté à l'autonomie communale, ce vote est venu rappeler la force que peut prendre un mouvement populaire.

Cinq ans après l'arrêté fédéral instituant des mesures urgentes en matière d'aménagement du territoire, dont l'application dans le canton de Vaud avait soulevé l'indignation des communes, une année après le refus de la première loi fédérale sur l'aménagement du territoire repoussée au nom du fédéralisme, ce scrutin à contre-courant est venu remettre en cause la répartition des compétences et indirectement l'autonomie communale (HAINARD 1978). S'inscrivant dans un courant centralisateur, le succès de l'initiative a démontré que des enjeux aussi puissants que la protection des sites étaient capables de provoquer une inversion des tendances. A Lavaux, l'aménagement ne se fera désormais plus du bas vers le haut. Pour la première fois, la défense de l'autonomie communale n'est pas parvenue à emporter l'adhésion de la majorité des Vaudois. Considérée pourtant d'une façon générale comme le niveau privilégé de la participation où la connaissance du territoire est la plus favorable à un aménagement rationnel, la commune a été sanctionnée pour avoir insuffisamment réagi devant une évolution jugée négative par la plus grande partie de l'électorat vaudois. L'histoire dira si cette remise en question de l'institution communale restera un fait isolé.

\section{Le plan de protection de Lavaux; une mesure d'exception}

Quelque fondées que soient les réserves que l'on peut faire à une organisation de l'espace confiée uniquement aux communes, elles ne sauraient selon nous justifier une généralisation de la politique adoptée à Lavaux.

Uniquement au nom du Beau, répondant à un souci de paysagiste, les mesures de protection ont une portée économique capitale puisqu'elles fixent de manière catégorique le destin viticole de cette région. Bien que la perspective d'une reconversion des cultures ne préoccupe actuellement pas l'esprit des vignerons, il est hors de doute qu'une évolution de la conjoncture pourrait mettre un jour en évidence la force de cette contrainte.

En neutralisant pratiquement l'extension de l'urbanisation, la loi va empêcher le vignoble de devenir résidentiel et de prendre la forme d'un parc urbain interdit au public, mais pourra-t-elle empêcher de lui conférer un aspect progressivement figé qui n'en ferait plus à la longue qu'un espace témoin désincarné?

Partielle, l'appropriation sociale de Lavaux n'est que celle d'une image. Aucune culture n'est plus exclusive, intolérante que la vigne et, par ailleurs, le plan n'a pas cherché à rendre plus facile l'accès du public au lac ni à ouvrir de nouvelles plages. C'est dire que le peuple vaudois s'est davantage offert un tableau qu'un espace de délassement, considérant que toute altération du site de Lavaux était une altération de sa propre image.

Etant donné la valeur symbolique de la région, comparable à nulle autre, la vertu du coup de semonce pour le reste du canton est singulièrement affaiblie. L'intervention pour l'exemple semble avoir frappé la seule région possible et cette situation d'exception a permis de masqueer quelques 
problèmes fondamentaux de l'aménagement du territoire.

A aucun moment par exemple, les méfaits de la plus-value foncière et de la rente différentielle, véritable entrave à une libération sociale dans l'espace, ne furent abordés. L'évocation du prix des terres n'intervint que pour estimer le coût global de la protection ou pour infléchir les décisions dans des cas particuliers au vu des risques d'indemnités à verser.

L'énumération de pareilles limites doit nous détourner de la tentation de généraliser une telle pratique qui se trouve en fait être contraire à un idéal démocratique. La protection des sites reste au niveau du sauvetage. Elle est aux antipodes d'une politique d'aménagement communautaire qui, se voulant libératrice, reposerait sur une anticipation sociale et chercherait à maîtriser l'inscription des actes humains dans l'espace en ayant constamment à l'esprit le respect de l'environnement.

\section{Bibliographie}

'A. BYRN Etude d'un plan directeur LAVAUX. F. BOLOGNINI Cahiers de l'aménagement régional A. GUIDETTI No 9. Office cantonal vaudois de l'urbanisme. Lausanne 1971.

DUBOIS, J.: Problèmes de l'encepagement au Pays de Vaud. Tirage à part de la Nouvelle Revue de Lausanne. s. d.

DUMMER, P. A.: «SAUVER LAVAUX» Mémoires et Documents 4 de l'Institut de Science Politique. Lausanne 1973.

HAINARD, F.: Ethnologie des sociétés rurales. Le vignoble de Lavaux (VD). Séminaire de troisième cycle d'ethnologie. Université de Fribourg, Genève, Lausanne, Neuchâtel, Berne. Neuchâtel 1978.

JAQUES, M.: La viticulture à Lavaux et les problèmes du sol. Office cantonal vaudois de l'Urbanisme. Lausanne 1970.

ONDE, H.: Une terre, ses origines, ses régions. Encyclopédie illustrée du Pays de Vaud. Vol. No 2, 1971.

RUFFY, V.: L'aménagement du territoire de deux communes vaudoises: Lutry et Savigny. Son évolution. Geographica Helvetica XIX, No 2, 1964.

URECH, J. D.: Lavaux. Etude d'aménagement régional. Plan directeur. Commission intercommunale d'urbanisme de Lavaux. Lausanne 1977.

WEBER, F.: Des montagnes à soulever. JEAN-JACQUES PAUVERT, Paris 1976.
- Exposé des motifs et projet de loi sur la protection de la région de Lavaux. (Automne 1977).

- Exposé des motifs et projet de loi sur le plan de protection de Lavaux. (Septembre 1978).

- Inventaire des paysages et des sites naturels d'importance nationale qui méritent d'être protégés. Ligue suisse pour la protection de la nature, Ligue suisse de sauvegarde du patrimoine national et Club alpin suisse. Deuxième édition revisée. Walter-Verlag AG, Olten, 1967.

- Inventaire cantonal des monuments naturels et des sites, approuvé le 16 août 1972 par le Conseil d'Etat.

- Plans d'extension cantonaux No 43 et 43 bis Saint-Saphorin 1948 et 1961.

- Plan d'extension cantonal No 45. Puidoux, 1950.

\section{Zusammenfassung}

Die Geschichte der Gegend des Lavaux und die seines Weinbaus waren bis zu Beginn dieses Jahrhunderts noch eng verbunden. Daraus hatte sich eine Landschaft von vollendeter Schönheit und Harmonie entwickelt. Bei der meisterhaften Ausnützung der landschaftlichen Gegebenheiten war nichts dem Zufall überlassen worden. So wurde das Lavaux zum Symbol für die Ausdauer seiner Bewohner, zum Symbol für ihre Beherrschung der Umwelt und ihren Sinn für Tradition.

Die Verstädterung im 20. Jahrhundert bedrohte die Eigentümlichkeit dieser Landschaft ernstlich. Von außen her begann sie mit ihren negativen Auswirkungen gegen das Herz des Lavaux vorzudringen. Obwohl die gesetzlichen Grundlagen zur Verfügung standen, wurde mangels politischer Entschlußkraft vorerst weder eine Eindämmung noch eine Kontrolle der in verheerendem Maß fortschreitenden Bautätigkeit angeordnet.

Ein Teil der ansässigen Bevölkerung gründete schließlich die Vereinigung "Sauver Lavaux», um dieser Entwicklung Einhalt zu gebieten. Anlaß dazu war ein Projekt der schützenswerten Gemeinde Villette. "Sauver Lavaux» legte eine Gesetzesinitiative vor, die angenommen wurde, was zur Ausarbeitung einer der strengsten Schutzpläne der Schweiz beitrug. Eine derart restriktive Raumplanungspolitik kann trotz ihren unbestreitbar positiven Auswirkungen nicht empfohlen werden. Einerseits schränkt sie die Verwirklichung zum voraus festgelegter sozialer Ziele zu stark ein, andererseits hemmt sie die Entwicklung eines bewußten, respektvollen Verhaltens gegenüber der Umwelt. 